\title{
Normalization of the Mathieu-Gauss optical beams
}

\author{
Julio C. Gutiérrez-Vega \\ Photonics and Mathematical Optics Group, Tecnológico de Monterrey, Monterrey, México 64849
}

Miguel A. Bandres

California Institute of Technology, Pasadena, California 91125

Received June 15, 2006; revised July 27, 2006; accepted July 27, 2006; posted August 8, 2006 (Doc. ID 71939); published December 13, 2006

\begin{abstract}
A series scheme is discussed for the determination of the normalization constants of the even and odd Mathieu-Gauss (MG) optical beams. We apply a suitable expansion in terms of Bessel-Gauss (BG) beams and also answer the question of how many BG beams should be used to synthesize a MG beam within a tolerance. The structure of the normalization factors ensures that MG beams will always be normalized independently of the particular normalization adopted for the Mathieu functions. In this scheme, the normalization constants are expressed as rapidly convergent series that can be calculated to an arbitrary precision. (C) 2006 Optical Society of America

OCIS codes: $260.1960,350.5500,140.3300,050.1960$.
\end{abstract}

\section{INTRODUCTION}

Mathieu beams constitute a complete and orthogonal family of nondiffracting optical beams that are solutions of the wave equation in elliptic coordinates. ${ }^{1-4}$ These beams are fundamental in the sense that any optical field can be expanded in terms of Mathieu beams with appropriate weight factors and spatial frequencies. ${ }^{5}$ Mathieu beams have been applied, for example, to photonic lattices, ${ }^{6}$ transfer of angular momentum using optical tweezers, ${ }^{7,8}$ and localized $X$ waves. ${ }^{9}$

Since ideal Mathieu beams are not square integrable, they cannot be normalized such that the integral of the intensities is unitary. In view of this, we reported, in a recent paper, a closed and elegant expression for the propagation of the so-called Mathieu-Gauss (MG) beams, ${ }^{10}$ i.e., Mathieu beams apodized by a Gaussian transmittance, which carry a finite power and can be generated experimentally to a very good approximation. ${ }^{11} \mathrm{MG}$ beams constitute one of the simplest approximations to the exact nondiffracting Mathieu beams, and their propagation properties have been studied in free space s.,11 $^{10}$ and through apertured $A B C D$ optical systems. ${ }^{12,13}$

Although the propagation characteristics of the MG beams are well understood, the normalization factors of these beams have not been calculated until now. The object of the present investigation is to remedy this deficiency. Though the transverse intensity distribution of the MG beams cannot be integrated directly in a closed form, we shall see that a suitable expansion in terms of BesselGauss (BG) beams can be applied with success to the problem, yielding the normalization constants. The analysis of convergence provides an upper bound for the number of terms required to compute the normalization constants within a given accuracy. In the course of this analysis, we also answer the related question of how many BG beams should be used to synthesize a MG beam within a tolerance. Knowledge of the normalization factors is necessary for the construction of helical MG beams whose even and odd components should transport the same power. ${ }^{10,11}$

\section{DEFINITION OF THE MATHIEU-GAUSS BEAMS}

We briefly describe MG beams to establish notation and to provide a reference point for necessary formulas. We commence by defining the elliptic coordinates with foci at $\left( \pm f_{0}, 0\right)$ as

$$
\begin{aligned}
& x=f_{0} \cosh \xi \cos \eta, \\
& y=f_{0} \sinh \xi \sin \eta,
\end{aligned}
$$

where $\xi \in[0, \infty)$ and $\eta \in[0,2 \pi)$ are the radial and angular elliptic coordinates, respectively. Since we are primarily interested in finding the normalization constants of the beams, it is convenient to work with the expressions of the $m$ th-order even and odd MG beams at the plane $z=0$, namely,

$$
\begin{aligned}
& \mathrm{MG}_{m}^{e}(\xi, \eta, z=0)=\mathcal{N}_{m}^{e} \exp \left(-\frac{r^{2}}{w_{0}^{2}}\right) \mathrm{Je}_{m}(\xi, q) \mathrm{ce}_{m}(\eta, q) \\
& \mathrm{MG}_{m}^{o}(\xi, \eta, z=0)=\mathcal{N}_{m}^{o} \exp \left(-\frac{r^{2}}{w_{0}^{2}}\right) \mathrm{Jo}_{m}(\xi, q) \operatorname{se}_{m}(\eta, q),
\end{aligned}
$$

where $m=0,1,2, \ldots$ for even beams, $m=1,2,3, \ldots$ for odd beams, and $\mathcal{N}_{m}^{e}$ and $\mathcal{N}_{m}^{o}$ are the normalization factors to 
be determined such that the integral of the intensities is unitary. The transverse distributions are described by the $m$ th-order even and odd radial Mathieu functions $\mathrm{Je}_{m}(\cdot)$ and $\mathrm{Jo}_{m}(\cdot)$, and the even and odd angular Mathieu functions $\operatorname{ce}_{m}(\cdot)$ and $\mathrm{se}_{m}(\cdot)$, respectively. ${ }^{15-18}$

In Eqs. (3), the expressions $U_{m}^{e}=\mathrm{Je}_{m}(\xi, q) \mathrm{ce}_{m}(\eta, q)$ and $U_{m}^{o}=\mathrm{Jo}_{m}(\xi, q) \operatorname{se}_{m}(\eta, q)$ represent the transverse fields of the even and odd ideal nondiffracting Mathieu beams and satisfy the two-dimensional Helmholtz equation,

$$
\left(\partial_{x x}+\partial_{y y}+k_{t}^{2}\right) U_{m}^{e, o}(\xi, \eta)=0
$$

in elliptic coordinates. ${ }^{1}$ The MG beams are characterized by the ellipticity parameter $q=k_{t}^{2} f_{0}^{2} / 4$, which carries information about the transverse wavenumber $k_{t}$ and the ellipticity of the coordinate system through $f_{0}$.

In the plane $z=0$, the transverse shape of the $\mathrm{MG}$ beams is characterized by a set of confocal elliptic and hyperbolic nodal lines. Several theoretical and experimental patterns have been reported elsewhere. ${ }^{10-12}$ Like BG beams, the MG beams of the form (3) constitute a complete family of paraxial fields in the sense that any Helmholtz-Gauss beam with the same waist size and spatial frequency can be expressed as a superposition of MG beams with the appropriate weight factors. ${ }^{10} \mathrm{BG}$ beams represent a special case of the MG beams when the focal distance of the elliptical coordinates reduces to zero.

\section{NORMALIZATION OF THE MATHIEU-GAUSS BEAMS}

In principle, the problem of finding the normalization factors of the MG beams reduces to the determination of the integral $\iint_{-\infty}^{\infty}\left|\mathrm{MG}_{m}\right|^{2} \mathrm{~d} S$ across the whole transverse plane $(x, y)$. Closed-form evaluation of this integral is not possible, fortunately, the problem can be solved by using the fact that MG beams may be expanded in terms of $B G$ beams. This fact is evident once the Bessel beams form a complete set of orthogonal solutions of the Helmholtz equation in cylindrical coordinates.

At the plane $z=0$, the even and odd BG beams are written as $^{14}$

$$
\mathrm{BG}_{m}^{e, o}(r, \theta)=\mathcal{K}_{m} \exp \left(-\frac{r^{2}}{w_{0}^{2}}\right) J_{m}\left(k_{t} r\right)\left\{\begin{array}{c}
\cos (m \theta) \\
\sin (m \theta)
\end{array}\right\},
$$

where $(r, \theta)$ are the polar coordinates, $J_{m}(\cdot)$ is the $m$ th order Bessel function, and $\mathcal{K}_{m}$ is a normalization constant. It is convenient to explicitly find the factor $\mathcal{K}_{m}$ in order to work with an orthonormal basis of BG beams. The evaluation of the condition $\int_{0}^{\infty} \int_{0}^{2 \pi}\left|\mathrm{BG}_{m}\right|^{2} r \mathrm{~d} r \mathrm{~d} \theta=1$ leads to

$$
\mathcal{K}_{m}=\frac{2 \exp (\sigma / 2)}{w_{0} \sqrt{\left(1+\delta_{m, 0}\right) \pi I_{m}(\sigma)}}
$$

where $I_{m}(\cdot)$ is $m$ th-order modified Bessel function of the first kind, $\delta_{i, j}$ is the Kronecker delta, and

$$
\sigma \equiv \frac{k_{t}^{2} w_{0}^{2}}{4}
$$

is a beam parameter, which relates the transverse beam oscillations characterized by $1 / k_{t}$ and the Gaussian width given by $w_{0}$.

\section{A. Normalization of the $\mathrm{MG}_{2 n}^{e}(\xi, \eta)$ Beam}

Before proceeding with the normalization of the MG beams, we write the Fourier series representation of the Mathieu functions $\operatorname{ce}_{m}(\cdot)$ and $\operatorname{se}_{m}(\cdot)$. The expansions fall into four classes, according to their symmetry or antisymmetry, at approximately $\eta=0$ and $\eta=\pi / 2$, namely,

$$
\begin{aligned}
\operatorname{ce}_{2 n}(\eta ; q) & =\sum_{j=0}^{\infty} A_{2 j} \cos (2 j \eta), \\
\operatorname{ce}_{2 n+1}(\eta ; q) & =\sum_{j=0}^{\infty} A_{2 j+1} \cos [(2 j+1) \eta], \\
\operatorname{se}_{2 n+2}(\eta ; q) & =\sum_{j=0}^{\infty} B_{2 j+2} \sin [(2 j+2) \eta], \\
\operatorname{se}_{2 n+1}(\eta ; q) & =\sum_{j=0}^{\infty} B_{2 j+1} \sin [(2 j+1) \eta],
\end{aligned}
$$

where $n=0,1,2, \ldots$, and the Fourier coefficients $A$ and $B$ are functions of $q$ and are characterized by a set of known three-term recurrence relations. ${ }^{15-17}$ The corresponding series for the radial Mathieu functions are obtained from Eqs. (8) by setting the change of variable $\eta \rightarrow i \xi$.

Several normalizations have been proposed for the functions $\operatorname{ce}_{m}(\cdot)$ and $\operatorname{se}_{m}(\cdot){ }^{16,18}$ The most adopted in the literature is the same as that for the cosine and sine functions, i.e., $\int_{0}^{2 \pi} \mathrm{ce}_{m}^{2}(\eta ; q) \mathrm{d} \eta=\int_{0}^{2 \pi} \mathrm{se}_{m}^{2}(\eta ; q) \mathrm{d} \eta=\pi$ for all real values of $q$. Under this normalization, the Fourier coefficients have the property,

$$
\sum_{j=0}^{\infty}\left(1+\delta_{j, 0}\right) A_{2 j}^{2}=\sum_{j=0}^{\infty} A_{2 j+1}^{2}=\sum_{j=0}^{\infty} B_{2 j+2}^{2}=\sum_{j=0}^{\infty} B_{2 j+1}^{2}=1 .
$$

To normalize the MG beams, we commence by writing an integral equation for the $2 n$ th-order even Mathieu functions ${ }^{15-17}$

$$
\mathrm{Je}_{2 n}(\xi, q) \mathrm{ce}_{2 n}(\eta, q)=C_{2 n} \int_{0}^{2 \pi} \operatorname{ce}_{2 n}(\phi) \exp \left[\mathrm{i} k_{t} r \cos (\phi-\theta)\right] \mathrm{d} \phi,
$$

where the multiplier $C_{2 n}$ is given by

$$
C_{2 n}=\frac{\mathrm{ce}_{2 n}(0 ; q) \mathrm{ce}_{2 n}(\pi / 2 ; q)}{2 \pi A_{0}} .
$$

From a physical point of view, Eq. (10) represents the expansion of the ideal nondiffracting Mathieu beam $U_{2 n}^{e}$ $=\mathrm{Je}_{2 n}(\xi, q) \mathrm{ce}_{2 n}(\eta, q)$ in terms of plane waves. ${ }^{1}$ As well known from the theory of nondiffracting beams, ${ }^{4}$ the angular spectrum of the beam reduces to a circular delta of radius $k_{t}$ in the $\mathbf{k}$ space, which (in this case) is modulated 
angularly by the function $\mathrm{ce}_{2 n}(\phi)$. It is important to remark that Eq. (10) is a fundamental identity that holds independently of the normalization scheme adopted for the Mathieu functions.

We now proceed by inserting the series representation (8a) into the right side of Eq. (10). Since the integral of the sum is equal to the sum of the integrals, we have

$$
\begin{aligned}
\mathrm{Je}_{2 n}(\xi, q) \mathrm{e}_{2 n}(\eta, q)= & C_{2 n} \sum_{j=0}^{\infty} A_{2 j} \int_{0}^{2 \pi} \cos (2 j \phi) \\
& \times \exp \left[\mathrm{i} k_{t} r \cos (\phi-\theta)\right] \mathrm{d} \phi .
\end{aligned}
$$

The evaluation of the integral can be expressed in closed form using Bessel functions, ${ }^{16}$ namely,

$$
\mathrm{Je}_{2 n}(\xi, q) \mathrm{ce}_{2 n}(\eta, q)=C_{2 n} \sum_{j=0}^{\infty} A_{2 j}\left[(-1)^{j} 2 \pi J_{2 j}\left(k_{t} r\right) \cos (2 j \theta)\right] .
$$

Equation (13) is, in fact, the expansion of the nondiffracting Mathieu beams in terms of the Bessel beams. We now multiply both sides of Eq. (13) by the Gaussian modulation and the unknown normalization factor $\mathcal{N}_{2 n}^{e}$ to construct the MG beam,

$$
\begin{aligned}
\mathrm{MG}_{2 n}^{e}(\xi, \eta)= & \mathcal{N}_{2 n}^{e} \exp \left(-\frac{r^{2}}{w_{0}^{2}}\right) \mathrm{Je}_{2 n}(\xi, q) \mathrm{ce}_{2 n}(\eta, q), \\
= & \mathcal{N}_{2 n}^{e} 2 \pi C_{2 n} \sum_{j=0}^{\infty}(-1)^{j} A_{2 j} \\
& \times \exp \left(-\frac{r^{2}}{w_{0}^{2}}\right) J_{2 j}\left(k_{t} r\right) \cos (2 j \theta) .
\end{aligned}
$$

Using the definition of the BG beams in Eq. (5), we obtain the expansion of the normalized MG beams in terms of normalized BG beams,

$$
\mathrm{MG}_{2 n}^{e}(\xi, \eta)=\mathcal{N}_{2 n}^{e} 2 \pi C_{2 n} \sum_{j=0}^{\infty} \frac{(-1)^{j} A_{2 j}}{\mathcal{K}_{2 j}} \mathrm{BG}_{2 j}^{e}(r, \theta) .
$$

The normalization constant $\mathcal{N}_{2 n}^{e}$ is determined by the stipulation that $\iint_{-\infty}^{\infty}\left|\mathrm{MG}_{2 n}^{e}\right|^{2} \mathrm{~d} A=1$. Taking into account that BG beams form an orthonormal basis, from Eq. (15), we readily get the following relation,

$$
\left(\mathcal{N}_{2 n}^{e} 2 \pi C_{2 n}\right)^{2} \sum_{j=0}^{\infty}\left(\frac{A_{2 j}}{\mathcal{K}_{2 j}}\right)^{2}=1 .
$$

Finally, substituting the values of $C_{2 n}$ and $\mathcal{K}_{m}$ into the above equation, we can formally write the normalization constant for the $\mathrm{MG}_{2 n}^{e}$ beam,

$$
\mathcal{N}_{2 n}^{e}=\frac{2 \exp (\sigma / 2)}{\sqrt{\pi} w_{0} \operatorname{ce}_{2 n}(0 ; q) \operatorname{ce}_{2 n}(\pi / 2 ; q)}\left[\frac{A_{0}}{\sqrt{\sum_{j=0}^{\infty}\left(1+\delta_{j, 0}\right) I_{2 j}(\sigma) A_{2 j}^{2}}}\right] .
$$

As expected, $\mathcal{N}_{2 n}^{e}$ is a function of the beam parameter $\sigma$ $=k_{t}^{2} w_{0}^{2} / 4$ and the ellipticity parameter $q=k_{t}^{2} f_{0}^{2} / 4$.

\section{B. Normalization of the Remaining Cases}

Analysis similar to that in the previous subsection leads to the normalization factors for the remaining $\mathrm{MG}_{2 n+1}^{e}$, $\mathrm{MG}_{2 n+2}^{o}$, and $\mathrm{MG}_{2 n+1}^{o}$ beams. For the $\mathrm{MG}_{2 n+1}^{e}$ beam,

$$
\mathrm{MG}_{2 n+1}^{e}=\mathcal{N}_{2 n+1}^{e} \exp \left(-\frac{r^{2}}{w_{0}^{2}}\right) \mathrm{Je}_{2 n+1}(\xi, q) \mathrm{ce}_{2 n+1}(\eta, q),
$$

we obtain

$\mathcal{N}_{2 n+1}^{e}$

$$
=\frac{2 \sqrt{q} \exp (\sigma / 2)}{\sqrt{\pi} w_{0} \operatorname{ce}_{2 n+1}(0 ; q) \mathrm{ce}_{2 n+1}^{\prime}(\pi / 2 ; q)}\left[\frac{A_{1}}{\sqrt{\sum_{j=0}^{\infty} I_{2 j+1}(\sigma) A_{2 j+1}^{2}}}\right],
$$

where the prime denotes the derivative of the function $\operatorname{ce}_{m}(\eta ; q)$ with respect to the variable $\eta$.

In the same way, the normalized odd MG beams are given below

$$
\begin{aligned}
& \mathrm{MG}_{2 n+2}^{o}=\mathcal{N}_{2 n+2}^{o} \exp \left(-\frac{r^{2}}{w_{0}^{2}}\right) \mathrm{Jo}_{2 n+2}(\xi, q) \mathrm{Se}_{2 n+2}(\eta, q), \\
& \mathrm{MG}_{2 n+1}^{o}=\mathcal{N}_{2 n+1}^{o} \exp \left(-\frac{r^{2}}{w_{0}^{2}}\right) \mathrm{Jo}_{2 n+1}(\xi, q) \mathrm{se}_{2 n+1}(\eta, q),
\end{aligned}
$$

where

$\mathcal{N}_{2 n+2}^{o}$

$$
=\frac{2 q \exp (\sigma / 2)}{\sqrt{\pi} w_{0} \mathrm{se}_{2 n+2}^{\prime}(0 ; q) \mathrm{se}_{2 n+2}^{\prime}\left(\frac{\pi}{2} ; q\right)}\left[\frac{B_{2}}{\sqrt{\sum_{j=0}^{\infty} I_{2 j+2}(\sigma) B_{2 j+2}^{2}}},\right.
$$

$\mathcal{N}_{2 n+1}^{o}$

$$
=\frac{2 \sqrt{q} \exp (\sigma / 2)}{\sqrt{\pi} w_{0} \mathrm{se}_{2 n+1}^{\prime}(0 ; q) \operatorname{se}_{2 n+1}\left(\frac{\pi}{2} ; q\right)}\left[\frac{B_{1}}{\sqrt{\sum_{j=0}^{\infty} I_{2 j+1}(\sigma) B_{2 j+1}^{2}}}\right] .
$$

Equations (17), (19), (22), and (23) are the main result of this study. The structure of these factors ensures that MG beams will always be normalized independently of the particular normalization adopted for the Mathieu functions.

\section{NUMERICAL DISCUSSION}

Having given the series expression for the normalization constants, one of the main questions to be answered is how many terms of the infinite summations in Eqs. (17), (19), (22), and (23) should be used in order to compute the 
normalization constants within a given accuracy. This question is equivalent to asking the number of BG beams required in Eq. (15) to synthesize a MG beam within a tolerance. To answer the question, consider that the sums have the general form

$$
S_{m}^{e, o}=\sum_{k=0}^{\infty} s_{k}=\sum_{k=0}^{\infty} D I_{k}(\sigma) A_{k}^{2}
$$

where $k=2 j=0,2,4, \ldots$ for sums in Eqs. (17) and (22), $k$ $=2 j+1=1,3,5, \ldots$ for sums in Eqs. (19) and (23), and $D$ $=1$ except for the sum in Eq. (17) where $D=1+\delta_{j, 0}$.

The standard practice is to truncate the series when the term we have just added is smaller in magnitude than some small times the magnitude of the sum thus far accumulated. In this direction, an upper bound for the number of terms can be estimated by finding the ratio between consecutive coefficients, i.e.,

$$
V_{k} \equiv\left|\frac{s_{k}}{s_{k-2}}\right|=\left|\frac{I_{k}(\sigma)}{I_{k-2}(\sigma)} \frac{A_{k}^{2}}{A_{k-2}^{2}}\right| .
$$

From calculus we know that if $\lim _{k \rightarrow \infty} V_{k}<1$ then the series (24) is absolutely convergent. In this case, approximations to the sum can be obtained to any desired precision by setting $V_{k}$ lower than a given tolerance.

To determine the ratio $V_{k}$, we commence by writing the known three-term recurrence relations among the Fourier coefficients $A_{k}$ and $B_{k}$ of the Mathieu functions, namely, ${ }^{15-17}$

$$
\left(a-k^{2}\right) A_{k}-q\left(A_{k-2}+A_{k+2}\right)=0,
$$

where $k \geqslant 4$ and $a$ is the eigenvalue of the Mathieu equation $\left[d_{\eta}^{2}+a-2 q \cos 2 \eta\right] \Phi(\eta)=0$. We remark that Eq. (26) holds for the four classes of Mathieu functions in Eqs. (8).

By defining the ratio between consecutive Fourier coefficients as $G_{k} \equiv A_{k} / A_{k-2}$, Eq. (26) becomes

$$
G_{k+2}+\frac{1}{G_{k}}=\frac{a-k^{2}}{q} .
$$

From this relation, we conclude that $G_{k+2}+1 / G_{k} \rightarrow-\infty$ monotonically as $k \rightarrow \infty$. Hence either $G_{k} \rightarrow-\infty$ or $G_{k} \rightarrow 0^{-}$. The option $G_{k} \rightarrow-\infty$ is inadmissible because this implies $\left|A_{k}\right|>\left|A_{k-2}\right|$, and the sum would be divergent. Consequently, $G_{k} \rightarrow 0^{-}$as $k \rightarrow \infty$, leading to the fact that the magnitude of the Fourier coefficients tend to zero monotonically, and their signs alternate as $k$ increases. Now, the ratio $G_{k}$ can easily be determined from Eq. (27) in the limit when $k$ is very large; in this case $G_{k+2} \rightarrow 0$ and $a$ $\ll k^{2}$, thus

$$
\left|G_{k}\right|_{k \rightarrow \infty} \equiv\left|\frac{A_{k}}{A_{k-2}}\right|_{k \rightarrow \infty} \sim \frac{q}{k^{2}} .
$$

Coming back to Eq. (25), to determine the convergence of the ratio $I_{k}(\sigma) / I_{k-2}(\sigma)$, we use the asymptotic expansion of the $k$ th-order modified Bessel function $I_{k}(\sigma)$ for large order, ${ }^{16}$
Table 1. Normalized Terms of the Series in Eqs. (19) and (23) ${ }^{a}$

\begin{tabular}{ccc}
\hline & $\begin{array}{c}S_{1}^{e} \\
\text { Eq. }(19)\end{array}$ & $\begin{array}{c}S_{1}^{o} \\
\text { Eq. }(23)\end{array}$ \\
\hline$s_{1} / s_{1}$ & 1 & 1 \\
$s_{3} / s_{1}$ & 4.5914 & $5.2390 \times 10^{-1}$ \\
$s_{5} / s_{1}$ & 3.3800 & $1.4593 \times 10^{-1}$ \\
$s_{7} / s_{1}$ & $9.4118 \times 10^{-1}$ & $2.2185 \times 10^{-2}$ \\
$s_{9} / s_{1}$ & $1.2313 \times 10^{-1}$ & $1.9025 \times 10^{-3}$ \\
$s_{11} / s_{1}$ & $8.4416 \times 10^{-3}$ & $9.5374 \times 10^{-5}$ \\
$s_{13} / s_{1}$ & $3.2625 \times 10^{-4}$ & $2.8955 \times 10^{-6}$ \\
$s_{15} / s_{1}$ & $7.5122 \times 10^{-6}$ & $5.5051 \times 10^{-8}$ \\
$s_{17} / s_{1}$ & $1.0775 \times 10^{-7}$ & $6.7598 \times 10^{-10}$ \\
\hline
\end{tabular}

${ }^{a} m=1, q=50, \sigma=80$.
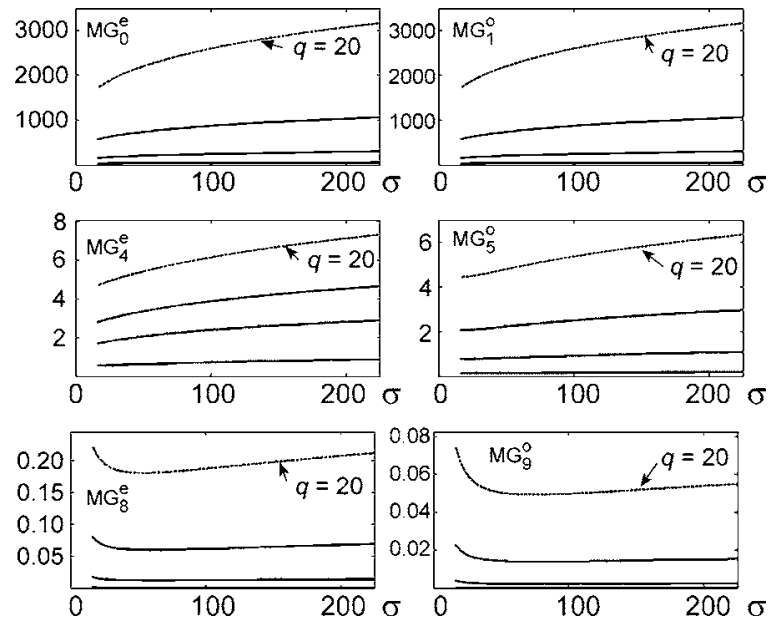

Fig. 1. Behavior of the normalization constants as a function of $\sigma$ for several orders $m$ and $q=5,10,15,20$.

$$
I_{k}(\sigma) \sim \frac{1}{\sqrt{2 \pi}} \frac{\exp \left(\sqrt{k^{2}+\sigma^{2}}\right)}{\left(k^{2}+\sigma^{2}\right)^{1 / 4}}\left(\frac{\sigma}{k+\sqrt{k^{2}+\sigma^{2}}}\right)^{k},
$$

where $k \geqslant \sigma$ must be satisfied. Note that for a fixed argument $\sigma>0$, the function $I_{k}(\sigma)$ is positive and decreases monotonically to zero as the order increases. Using Eq. (29) can demonstrate that the ratio $I_{k}(\sigma) / I_{k-2}(\sigma)$ may be approximated for large $k$ by

$$
\left.\frac{I_{k}(\sigma)}{I_{k-2}(\sigma)}\right|_{k \rightarrow \infty} \sim \frac{\sigma^{2}}{4 k^{2}} .
$$

Collecting the partial results in Eqs. (28) and (30), the ratio $V_{k}$ for large $k$ in Eq. (25) is found to be

$$
\left|V_{k}\right|_{k \rightarrow \infty} \equiv\left|\frac{s_{k}}{s_{k-2}}\right|_{k \rightarrow \infty} \sim \frac{\sigma^{2} q^{2}}{4 k^{6}}
$$

where $k=2 j$ for the sums in Eqs. (17) and (22) and $k=2 j$ +1 for the sums in Eqs. (19) and (23).

Equation (31) is of practical value for estimating how many terms of the series (24) do we need to add to guarantee a specified accuracy. Let $K$ be this number of required terms, if we set a tolerance (tol) for the ratio between consecutive coefficients, then from Eq. (31), we find 


$$
K=\operatorname{ceil}\left[\frac{1}{2}\left(\frac{\sigma^{2} q^{2}}{4 \text { tol }}-\varrho\right)^{1 / 6}\right]
$$

where the function ceil $(x)$ returns the smallest integer greater than or equal to $x$, and $\varrho=0$ for $\mathcal{N}_{2 n}^{e}, \mathcal{N}_{2 n+2}^{o}$, and $\varrho=1$ for $\mathcal{N}_{2 n+1}^{e}, \mathcal{N}_{2 n+1}^{e}$. It is instructive to illustrate the

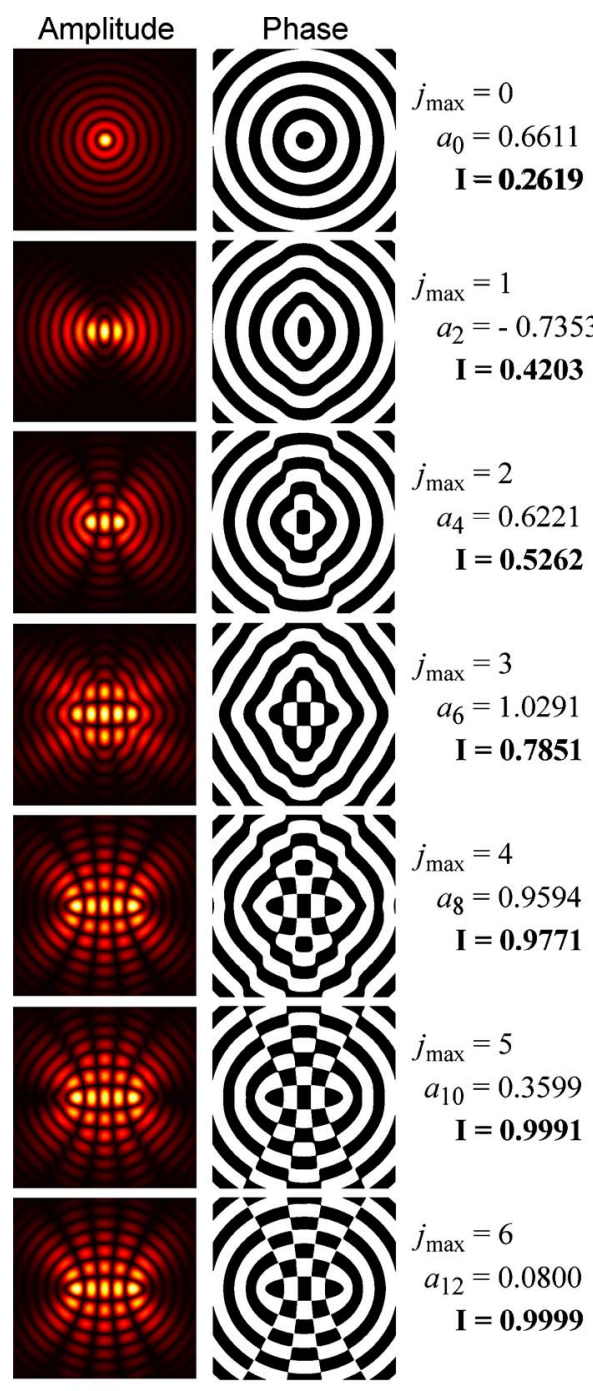

(a)

(b)

Fig. 2. (Color online) (a) Amplitude and (b) phase of the reconstruction of a $\mathrm{MG}_{4}^{e}(\xi, \eta ; q=25)$ beam using finite superpositions of BG beams. $j_{\max }$ denotes the number of BG beams used to build up the MG beam.

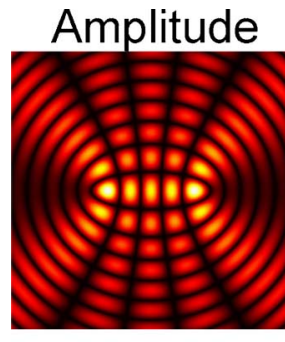

(a)

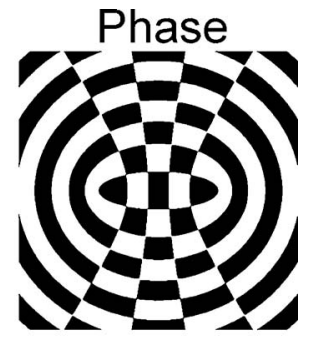

(b)
Fig. 3. (Color online) Pure Mathieu nondiffracting beam corresponding to the MG beam shown in Fig. 2. convergence of the series (24) with a concrete example. In Table 1, we include the first terms $s_{k}$ for the sums in Eqs. (19) and (23) with $m=1, q=50$, and $\sigma=80$. For comparison purposes, we have normalized the terms with respect to the first term of the series. As we can see, the series are rapidly convergent. With the same aim, the behavior of the normalization constants as a function of the beam parameter $\sigma$ is depicted in Fig. 1 for several orders $m$ and $q=\{5,10,15,20\}$. For all plots, the dashed curve corresponds to $q=20$. The curves were determined by calculating Eqs. (17), (19), (22), and (23) for a range of $\sigma$ keeping in mind that the paraxial approximation requires that $\sigma$ $\gg 1$ as discussed in Ref. 10.

We also remark that Eq. (28) provides an upper bound for the number of coefficients we are willing to use in the computation of the Mathieu functions using the series (8). If the series is truncated at the $K$ th term, then an upper bound for the roundoff error can be computed using the known result from calculus that if Eq. (24) is an alternating series with limit $S$ whose terms decrease in magnitude, then $S$ and the $K$ th partial sum $S_{K}=\sum_{k=0}^{K} s_{k}$ differ by less than the magnitude of the $(K+1)$ th term, that is, $\mid S$ $-S_{K}|\leqslant| s_{K+1} \mid$.

As a final illustration of the series formalism developed in Section 3, in Fig. 2, we show the construction of a $\mathrm{MG}_{4}^{e}$ $(\xi, \eta ; q=25)$ beam using a finite superposition of $\mathrm{BG}$ beams. For each row in the figure, the pattern is calculated using $j_{\max }$ terms according to the general series [see Eq. (14b)] given by

$$
\operatorname{MG}_{4}^{e}(\xi, \eta)=\exp \left(-\frac{r^{2}}{w_{0}^{2}}\right) \sum_{j=0}^{j_{\max }} a_{2 j} J_{2 j}\left(k_{t} r\right) \cos (2 j \theta)
$$

where $a_{2 j}$ are the series coefficients, also shown in Fig. 1. As expected, the first term provides the contribution of the fundamental $J_{0} \mathrm{BG}$ beam. It is interesting to see how the initial circular shape gradually tends to the typical elliptic shape of the even MG beam as more terms are taken into account in the summation. For each row in Fig. 2 , the power of the beam is calculated with $I$ $=\iint_{-\infty}^{\infty}\left|\mathrm{MG}_{4}^{e}\right|^{2} \mathrm{~d} A$. Note that, in this case, only seven terms are needed to ensure that the beam be practically synthesized and, moreover, normalized. Finally, for comparison purposes, in Fig. 3, we include the transverse shape of the corresponding pure Mathieu nondiffracting beam. Comparing Figs. 2 and 3, we see the effect of the Gaussian apodization of the shape of the Mathieu beam.

\section{CONCLUSIONS}

We have shown that the normalization constants for the four classes of MG beams can be expressed in a calculable rapidly convergent series and thus can be computed to an arbitrary precision. The structure of the factors ensures that MG beams will always be normalized independently of the particular normalization adopted for the Mathieu functions. It was possible to give an estimation of the number of required terms to guarantee a specified accuracy. We also answered the question of how many $B G$ beams are required to build up a MG beam. The proce- 
dure followed in this paper can also be applied to find the normalization constants of other kinds of beams expressible in terms of BG beams.

\section{ACKNOWLEDGMENTS}

This research was partially supported by the Consejo Nacional de Ciencia y Tecnología of México grant 42808, and by the Tecnológico de Monterrey Research Chair in Optics grant CAT-007. M. A. Bandres acknowledges support from Secretaría de Educación Pública de México.

Corresponding author J. C. Gutiérrez-Vega's e-mail address is juliocesar@itesm.mx.

\section{REFERENCES}

1. J. C. Gutiérrez-Vega, M. D. Iturbe-Castillo, and S. ChávezCerda, "Alternative formulation for invariant optical fields: Mathieu beams," Opt. Lett. 25, 1493-1495 (2000).

2. J. C. Gutiérrez-Vega, M. D. Iturbe-Castillo, G. A. Ramírez, E. Tepichín, R. M. Rodríguez-Dagnino, S. Chávez-Cerda, and G. H. C. New, "Experimental demonstration of optical Mathieu beams," Opt. Commun. 195, 35-40 (2001).

3. C. A. Dartora, M. Zamboni-Racheda, K. Z. Nobrega, E. Recami, and H. E. Hernández-Figueroa, "General formulation for the analysis of scalar diffraction-free beams using angular modulation: Mathieu and Bessel beams," Opt. Commun. 222, 75-80 (2003).

4. Z. Bouchal, "Nondiffracting optical beams: physical properties, experiments, and applications," Czech. J. Phys. 53, 537-578 (2003).

5. A. Chafiq, Z. Hricha, and A. Belafhal, "Paraxial propagation of Mathieu beams through an apertured ABCD optical system," Opt. Commun. 253, 223-230 (2005).
6. Y. V. Kartashov, A. A. Egorov, V. A. Vysloukh, and L. Torner, "Shaping soliton properties in Mathieu lattices," Opt. Lett. 31, 238-240 (2006).

7. S. Chávez-Cerda, M. J. Padgett, I. Allison, G. H. C. New, J. C. Gutiérrez-Vega, A. T. O'Neil, I. MacVicar, and J. Courtial, "Holographic generation and orbital angular momentum of high-order Mathieu beams," J. Opt. B: Quantum Semiclassical Opt. 4, S52-S57 (2002).

8. C. López-Mariscal, J. C. Gutiérrez-Vega, G. Milne, and K. Dholakia, "Orbital angular momentum transfer in helical Mathieu beams," Opt. Express 14, 4182-4187 (2006).

9. C. A. Dartora and H. E. Hernández-Figueroa, "Properties of a localized Mathieu pulse," J. Opt. Soc. Am. A 21, 662-667 (2004).

10. J. C. Gutiérrez-Vega and M. A. Bandres, "Helmholtz-Gauss waves," J. Opt. Soc. Am. A 22, 289-298 (2005).

11. C. López-Mariscal, M. A. Bandrés, and J. C. GutiérrezVega, "Observation of the experimental propagation properties of Helmholtz-Gauss beams," Opt. Eng. (Bellingham) 45, 068001 (2006).

12. A. Chafiq, Z. Hricha, and A. Belafhal, "A detailed study of Mathieu-Gauss beams propagation through an apertured $A B C D$ optical system," Opt. Commun. 265, 594-602 (2006).

13. M. Guizar-Sicairos and J. C. Gutiérrez-Vega, "Generalized Helmholtz-Gauss beam and its transformation by paraxial optical systems," Opt. Lett. 31, 2912-2915 (2006).

14. F. Gori, G. Guattari, and C. Padovani, "Bessel-Gauss beams," Opt. Commun. 64, 491-495 (1987).

15. N. W. McLachlan, Theory and Applications of Mathieu Functions (Oxford, 1951).

16. M. Abramowitz and I. Stegun, Handbook of Mathematical Functions (Dover, 1964).

17. I. S. Gradshteyn and I. M. Ryzhik, Table of Integrals, Series, and Products (Academic, 2000).

18. J. C. Gutiérrez-Vega, R. M. Rodríguez-Dagnino, M. A. Meneses-Nava, and S. Chávez-Cerda, "Mathieu functions, a visual approach," Am. J. Phys. 71, 233-242 (2003). 